\title{
Evidence of the causal role of human papillomavirus type 58 in an oropharyngeal carcinoma
}

Lorena Baboci ${ }^{1,2,3}$, Paolo Boscolo-Rizzo ${ }^{4}$, Dana Holzinger ${ }^{1}$, Roberta Bertorelle ${ }^{3}$, Lorena Biasini ${ }^{3}$, Angelika Michel ${ }^{1}$, Markus Schmitt ${ }^{7}$, Giacomo Spinato ${ }^{5}$, Rossana Bussani ${ }^{6}$, Laia Alemany ${ }^{7}$, Giancarlo Tirelli ${ }^{5}$, Maria Cristina Da Mosto ${ }^{4}$, Annarosa Del Mistro ${ }^{3 *}$ and Michael Pawlita ${ }^{1}$

\begin{abstract}
Persistent human papillomavirus infection (HPV) is recognized as an important etiologic factor for a subset of head and neck squamous cell carcinomas (SCC), especially those arising from the oropharynx. Whereas HPV16 accounts for the majority of HPV DNA-positive oropharyngeal SCC, infections with other mucosal high-risk HPV types are quite rare and biological data demonstrating their causal involvement are insufficient. Here we present the first case of an oropharyngeal SCC driven by HPV type 58. A 69-year-old Caucasian woman presented with an enlarged and firm left tonsil. A computed tomography scan showed a left tonsillar mass, extending to the soft palate and the glossotonsillar sulcus. The patient underwent extended radical tonsillectomy and ipsilateral selective neck dissection. Pathology confirmed an infiltrating, poorly differentiated SCC of the left tonsil with node metastasis (pT2N1). Adjuvant external beam radiation therapy (60 Grays (Gy)) was administered. After 1 year of follow-up, the patient is well with no evidence of cancer recurrence. HPV analyses of the tumor tissue by BSGP5+/6+-PCR/MPG, targeting 51 mucosal HPV types, showed single positivity for HPV type 58. Presence of HPV58 E6*I RNA demonstrated biological activity of the virus in the tumor tissue, and presence of serum antibodies to HPV58 oncoproteins E6 and E7 indicated presence of an HPV58-driven cancer. Overexpression of cellular protein $\mathrm{p} 16^{\mathrm{INK} 4 \mathrm{a}}$ and reduced expression of $\mathrm{pRb}$, two cellular markers for HPV-induced cell transformation, were observed. Exons 4-10 of TP53 showed no mutations or polymorphisms. The presence of HPV58 as single HPV infection in combination with a broad variety of direct and indirect markers of HPV transformation provides comprehensive evidence that this oropharyngeal SCC was driven by HPV58.
\end{abstract}

Keywords: HPV58, Head and neck squamous cell carcinoma, HPV carcinogenesis, HPV E6 and E7, HPV antibody, p16 $6^{\text {INK4a }}$, pRb, p53

\section{Background}

Oropharyngeal squamous cell carcinomas (OPSCC) are categorized as head and neck squamous cell carcinoma (HNSCC) together with squamous cell carcinoma of the oral cavity, larynx and hypopharynx. OPSCC account for approximately 50,000 incident cases [1,2], and together with hypopharyngeal squamous cell carcinomas they account for about $1.1 \%$ of all malignancies worldwide [3]. Tobacco smoking and alcohol consumption are recognized as major risk factors but infection with Human

\footnotetext{
* Correspondence: annarosa.delmistro@ioveneto.it

${ }^{3}$ Veneto Institute of Oncology IOV - IRCCS, Immunology and Molecular Oncology Unit, Via Gattamelata, 64, Padua 35128-I, Italy

Full list of author information is available at the end of the article
}

papillomaviruses (HPV) has been identified as a causal factor for an increasing number of OPSCC, particularly in Waldeyer's tonsillar ring $[4,5]$.

Among the 51 mucosal HPV types known so far, 12 have been classified as carcinogenic (class I) for cervical cancer (CxCa) [6]. While HPV type 16 is the most prevalent type in $\mathrm{CxCa}$ worldwide $(61 \%)$, the other carcinogenic types, i.e. HPV18, 31, 33, 35, 39, 45, 51, 52, 56, 58 and 59 (here referred to as non-HPV16 types) are responsible for approximately another $33 \%$ of $\mathrm{CxCa}$, with HPV58 specifically accounting for $2 \%$ of $\mathrm{CxCa}$. HPV58 in cervical cancer has the highest prevalence in Asia (4\%), followed by North- and South-America (2\% each), Europe (1\%) and Africa $(<1 \%)$ [7].

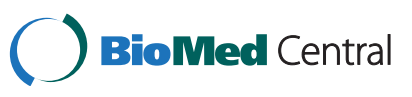


In contrast to $\mathrm{CxCa}$, an even larger majority of $\mathrm{HPV}$ DNA-positive OPSCC are associated with HPV16 (89\% 97\%), and DNA of other carcinogenic non-HPV16 types has been detected only rarely in OPSCC tissues [8-10]. A recent metanalysis of HPV DNA prevalence in head and neck cancers (Ndiaye $\mathrm{C}$, Alemany $\mathrm{L}$ et al., in preparation) identified 11 (0.8\%) HPV58 DNA positives among a total of 1466 HPV DNA positive oropharyngeal cancer cases that had been analysed for presence of HPV58 DNA [5,11-15].

Intriguingly, only a subset of HPV16 DNA-positive OPSCC display HPV16 carcinogenic activity in the tumor tissue, i.e. are HPV-driven (HPV DNA-positive RNA-positive) OPSCC, particularly in populations with low HPV DNA prevalence in OPSCC such as Western Europe. This indicates that presence of HPV DNA alone is not sufficient proof for causal involvement of any HPV DNA found in an OPSCC tissue. For non-HPV16 types found in OPSCC molecular proof of causality is largely lacking.

Over the last 25 years a rather detailed model of HPV-driven transformation of human tumor cells has been established. Truly HPV-transformed tumor cells contain at least one viral genome copy per cell and express the viral oncogenes E6 and E7. Interaction of the E6 and E7 oncoproteins with key regulators of cell cycle and apoptosis leads to upregulation of cellular protein p16 ${ }^{\mathrm{INK} 4 \mathrm{a}}$ and downregulation of tumor suppressor proteins $\mathrm{pRb}$ and $\mathrm{p} 53$. In patients with invasive HPV-driven cervical, penile and oropharyngeal SCC overexpression of E6 and E7 oncoproteins frequently leads to strong antibody responses against these viral proteins [4,16-24].

This model has been extensively verified in cervical carcinoma for all HPV types classified by IARC/WHO as carcinogenic, probably and possibly carcinogenic [25]. It has also been fully or partially verified for HPV16 in squamous cell carcinoma of the oropharynx $[8,26]$, larynx [27], oral cavity [28], penis [17] and anus [29].

Here, we present the first case of an OPSCC driven by HPV58, a carcinogenic HPV type with low prevalence in $\mathrm{CxCa}$, as demonstrated by a broad variety of HPV transformation markers. The present case is part of an ongoing prospective multicentric study of head and neck cancer patients from North-East Italy.

\section{Case presentation}

\section{Clinical data}

A 69-year-old Caucasian woman presented with a 2 months history of foreign-body sensation in the throat. She had a negative history for tobacco and alcohol use. The patient underwent regular gynecological examinations and cytological cervical cancer screenings until the age of 64, and no HPV-associated genital lesions or cytological alterations were found. No anogenital tumor had become symptomatically apparent since then and extending also into the follow-up period after treatment of the index throat condition. The patient got infected with hepatitis B virus (HBV) by blood transfusion at the age of 26 , and had been treated for lichen ruber planus in the oral cavity at the age of 34 . The ENT (Ear Nose and Throat) physical examination revealed an enlarged left tonsil without ulceration. On palpation, the left tonsil was found to be firm to hard in consistency. She did not have any palpable cervical lymphadenopathy. A computed tomography (CT) scan with contrast of the neck confirmed a left tonsillar lesion of $20 \times$ $23 \times 26 \mathrm{~mm}$ in dimension, extending up to the soft palate, without crossing midline, and down to the glossotonsillar sulcus, and revealed two homogeneously enhancing lymph nodes, with maximum transverse diameters of $13 \mathrm{~mm}$, in the left submandibular triangle. Baseline investigations including complete and differential blood cell count, serum electrolytes, liver and renal function tests, electrocardiogram, and X-ray chest, were well within normal limits. The patient underwent wedge biopsy of the left tonsillar mass. Pathologic review revealed the presence of a poorly differentiated SCC with basaloid features.

The patient underwent left side mandibulotomy, extended radical tonsillectomy and ipsilateral selective neck dissection (levels I-II-III-IV). The surgical defect was reconstructed with a microvascular ulnar forearm flap. Final pathology confirmed an infiltrating, poorlydifferentiated SCC of the left tonsil, with tumor measuring $28 \mathrm{~mm}$ in greatest dimension and a thickness of $14 \mathrm{~mm}$, abundant comedo-type necrosis and the presence of both vascular and perineural invasion. Histopathological examination of the neck dissection tissue showed an enlarged lymph node with intracapsular metastasis at level II (pT2N1). To minimize the risk of locoregional recurrence the treatment was completed with adjuvant external beam radiation therapy (EBRT), which started 7 weeks after surgery. Radiation treatment targeted the tonsillar bed and the left cervical region. A dose of 60 Gy was delivered with conventional fractionation (2 Gy/fraction, once daily, five times weekly). The HPV molecular data were not available at the time of clinical diagnosis and tumor treatment. After 1 year of follow-up, the patient is well with no evidence of recurrent cancer.

\section{Molecular analyses}

Analyses were performed in fresh-frozen tumor biopsy, formalin-fixed paraffin-embedded (FFPE) tumor tissue and serum. All samples were collected at the time of initial tumor diagnosis before surgery and radiotherapy.

Genomic DNA was extracted from sections of freshfrozen and FFPE tissues following previously described procedures $[8,25,30]$. More than $80 \%$ of the cells in hematoxylin eosin-stained adjacent sections were neoplastic. 
HPV genotyping was performed by BSGP5+/6+ -PCR/ MPG [31-33], an assay capable of amplifying about 150 base pairs (bp) of the L1 gene of 51 HPV mucosal types, followed by hybridization to type-specific probes on fluorescent suspension array beads. It includes detection of the human $\beta$-globin gene as DNA quality control as well as internal PCR and hybridization controls. Sample DNA was also analyzed by MY09/11-PCR amplification followed by both Restriction Fragment Length Polymorphism (RFLP) analysis and direct sequencing [34].

Genomic DNA extracted from both fresh-frozen and FFPE tumor tissues contained HPV58 as single mucosal HPV infection, as shown by both mucosal HPV genotyping methods.

Total RNA was obtained from FFPE tissue sections using the Pure-Link FFPE Total RNA Isolation Kit (Invitrogen, Carlsbad, CA) following the manufacturer's protocol. Viral RNA expression was analyzed by E6*I reverse transcription (RT)-PCR [25]. Briefly, the HPV type-specific assay generates $70 \mathrm{bp}$ long cDNA amplicons across the E6*I splice site that are subsequently detected by hybridization to type-specific and splice site-specific probes on fluorescent suspension array beads. A duplex assay was performed for RNA of HPV16 and the cellular housekeeping gene ubiquitin $\mathrm{C}(\mathrm{UbC})$, and a singleplex assay for HPV58. The sample was positive for the HPV58 E6*I and UbC transcripts and negative for the HPV16 E6*I transcript.

The HPV antibody status was analyzed by a glutathione S-transferase (GST) capture immunosorbent assay using as antigens full-length HPV proteins bacterially expressed as GST fusion proteins [35,36] in combination with fluorescent suspension array beads as previously described $[4,37,38]$. Antibodies to the E6 and E7 oncoproteins of high-risk HPV types 16, 18, 31, 33, 45, 52 and 58 and low-risk types 6 and 11 were quantified. Positivity was high for E6 and E7 of HPV58. Weaker signals were observed for E7 of the closely related HPV types 31 and 33 and are interpreted as cross-reactivity with the HPV58 E7 protein (Figure 1).

The expression level of the cellular proteins $\mathrm{p} 16^{\mathrm{INK} 4 \mathrm{a}}$ and $\mathrm{pRb}$, markers for HPV transformation, were evaluated by immunohistochemistry (IHC). The monoclonal antibodies CINtec (V-kit, MTM laboratories, Heidelberg, Germany) and NCL-RB (Novocastra, Newcastle, UK), were used for $\mathrm{p} 16^{\mathrm{INK} 4 \mathrm{a}}$ and $\mathrm{pRb}$, respectively. Well characterized sections from $\mathrm{CxCa}$ and a healthy mucosa were used in each staining batch as reference for scoring of the protein expression levels for both markers. IHC was evaluated independently by two investigators. The tumor cells of the sections showed strong nuclear and diffuse cytoplasmic staining of p16 ${ }^{\mathrm{INK} 4 \mathrm{a}}(>95 \%)$ and low staining of $\mathrm{pRb}(<25 \%)$, while proliferating basal and parabasal cells of the tumor-adjacent mucosa showed low p16 ${ }^{\mathrm{INK} 4 \mathrm{a}}(<5 \%)$ and high pRb (>25\%) expression (Figure 2).

TP53 mutation status was determined in genomic DNA isolated from the fresh-frozen biopsy. Exons 4-10 were amplified using specific primer pairs for each exon as described in the IARC protocol [39]. Amplification products were sequenced using the ABI PRISM ${ }^{\circ}$ 3730XL Genetic Analyzer (Applied Biosystems, Foster City, CA). No mutations or polymorphisms were observed.

\section{Discussion}

HPV-driven OPSCC appears to represent a tumor entity distinct from HPV-negative OPSCC. Patients with HPV-driven OPSCC in comparison to patients with HPV-negative OPSCC have a better outcome and overall survival, independent of treatment regimen [40,41]. These tumors also have been shown to contain fewer overall somatic mutations/genetic changes [42]. Furthermore, while in HPV-negative OPSCC tumor suppressor TP53 mutations are present in up to $100 \%$, such mutations are absent in HPV-positive OPSCC [42-44]. It therefore appears to be clinically important for prognosis and potentially for choice of treatment options to determine the HPV-status of OPSCC cases.

While for HPV16, the HPV-type most frequently found in HPV DNA-positive OPSCC, a transforming role in the pathogenesis of a subgroup of OPSCC has been thoroughly demonstrated $[8,16,45]$, this evidence is largely lacking for non-16 HPV types that are found in only $3-14 \%$ of OPSCC by HPV genotyping studies applying sensitive PCR techniques $[9,10]$. However, as demonstrated for HPV16 in several studies, presence of HPV DNA alone is not sufficient evidence for causal involvement of the HPV type found [8,22,45-47].

The HPV58-positive OPSCC case we describe here is, to our knowledge, the first case for which ample molecular and serological evidence is provided to demonstrate causal involvement of this rare high-risk HPV type in the pathogenesis of this carcinoma. In addition to the presence of HPV DNA in the tumor tissue, we demonstrate (i) presence of HPV58 E6\% I RNA encoding the viral oncoprotein E7, which in HPV58-positive cervical carcinoma is a consistent marker of biological activity of the virus [27]; (ii) strong serum antibody response to both HPV58 oncoproteins E6 and E7; HPV type concordant E6 and E7 double antibody positivity has been demonstrated to be highly associated and specific for invasive $\mathrm{HPV}$-associated cancers with an extremely low prevalence of about $0.1 \%$ in tumor-free individuals $[4,18,21]$ and to be strongly associated (odds ratios of 44 to 180) with invasive cancer of the cervix [20], the penis [17] and the upper aerodigestive tract [4,18,21]; (iii) overexpression of $\mathrm{p} 16^{\mathrm{INK} 4 \mathrm{a}}$ and reduced expression of $\mathrm{pRb}$, cellular markers for HPV transformation 


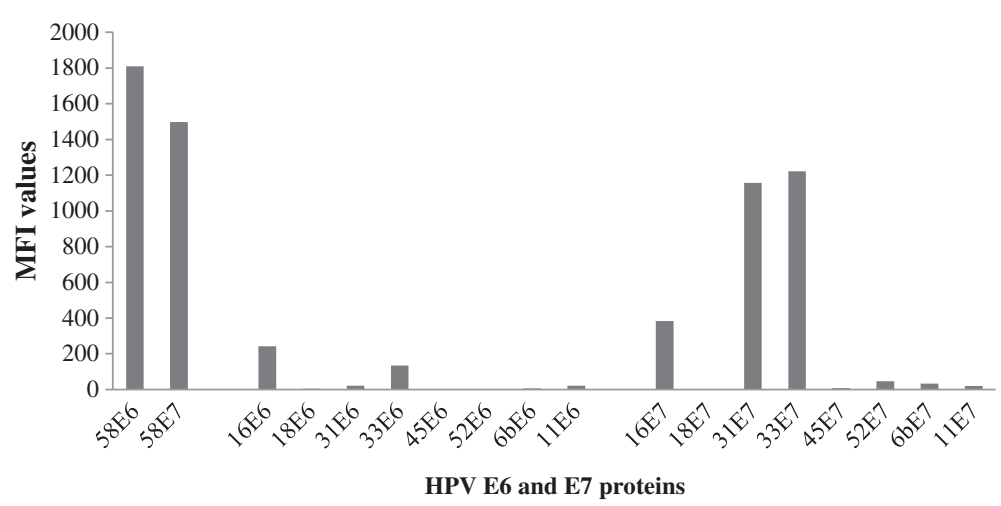

Figure 1 E6 and E7 antibodies status for HPV58 and other HPV types (16, 18, 31, 33, 45, 52, 6 and 11). MFI (Mean Fluorescence Intensity) values (y axis) are shown for the early proteins E6 and E7 of HPV58 and other HPV types $(16,18,31,33,45,6,11)$ ( $x$ axis). Left: E6 and E7 MFI values for HPV58; Center and right: the MFI values for the other HPV types tested, grouped as E6 and E7, respectively.

strongly associated with HPV16-transformed OPSCC [26] and also HPV58-transformed cervical cancers $[25,48]$, and finally (iv) wildtype sequence of TP53, a marker strongly associated with HPV16-positive OPSCC [42]. We further excluded the possibility of HPV16 involvement by demonstrating absence of HPV16 DNA, of HPV16 E6"I RNA and of HPV16 E6 and E7-specific antibodies. However, due to the rare involvement of HPV58 in HNSCC, multicentric studies are needed to evaluate the survival of the patient(s) to confirm the better prognosis like it was shown for HPV16-positive cases.

\section{Conclusion}

This HPV58-driven oropharyngeal carcinoma is the first well-documented case of HPV transformation in the oropharynx by a non-16 HPV-type.
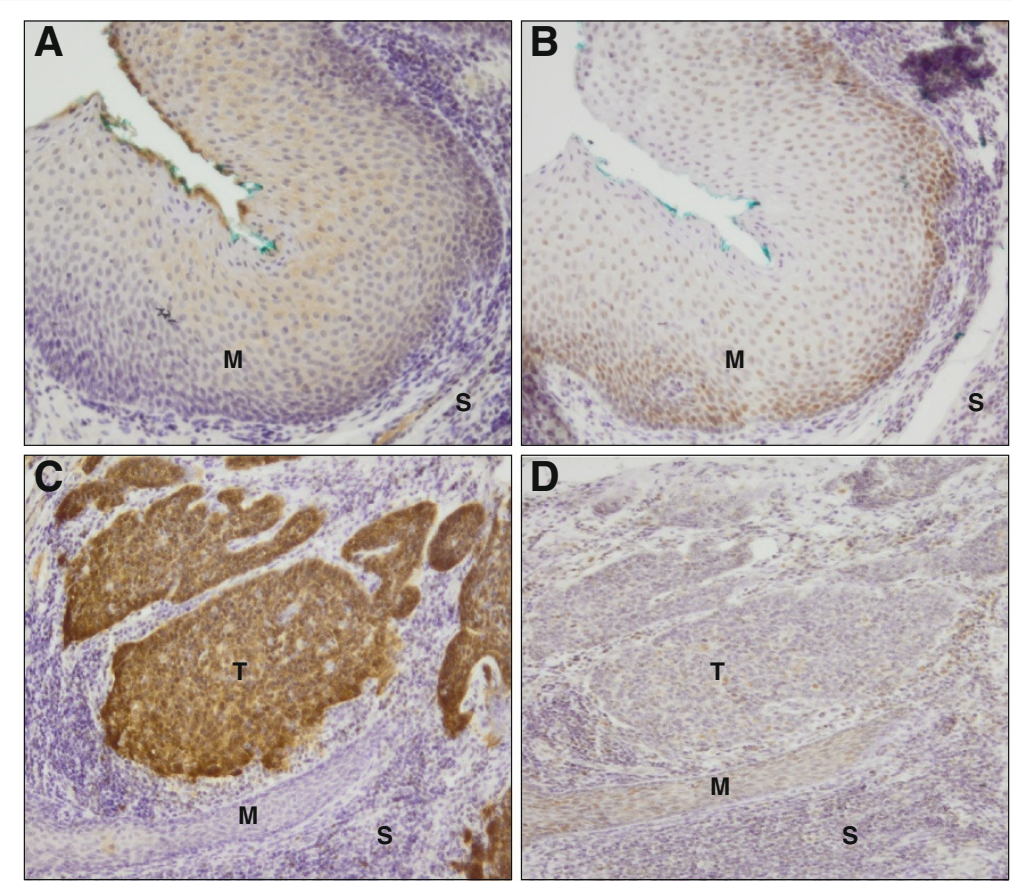

Figure 2 Immunohistochemistry of cellular proteins $\mathbf{p} 16^{\mathbf{I N K} 4 \mathrm{a}}$ and $\mathbf{p R b}$. A and $\mathbf{C}$, p16 $6^{\mathrm{INK} 4 \mathrm{a}}$ expression in normal tumor-adjacent mucosa (M) and in tumor (T) tissue, respectively. B and D, pRb expression in normal tumor-adjacent mucosa and in tumor tissue, respectively. S, stroma. Original magnification 10x. p16 ${ }^{\text {INK4a }}$ low in the normal tumor-adjacent mucosa (blue-stained nuclei (A)), high in the tumor (brown stained nuclei (C)). pRb high in normal tumor-adjacent mucosa (brown-stained nuclei (B)), low in the tumor (blue-stained nuclei (D)). 


\section{Consent}

Written informed consent was obtained from the patient for publication of this Case Report and any accompanying images. A copy of the written consent is available for review by the Editor-in-Chief of this journal.

\section{Abbreviations \\ CxCa: Cervical carcinoma; ENT: Ear, nose and throat; FFPE: Formalin-fixed paraffin-embedded; GST: Gluthatione S-transferase; Gy: Gray; HPV: Human papillomavirus; IARC: International agency for research on cancer; IHC: Immunohistochemistry; OPSCC: Oropharyngeal squamous cell carcinoma; PCR: Polymerase chain reaction; pRb: Retinoblastoma protein; UbC: Ubiquitin C; WHO: World Health Organization.}

\section{Competing interests}

The authors L. Baboci, D. Holzinger, P. Boscolo-Rizzo, A. Del Mistro, G. Spinato, R. Bertorelle, L. Biasini, A. Michel, R. Bussani, L. Alemany, G. Tirelli and M.C. Da Mosto disclosed no potential conflicts of interest. M. Schmitt and M. Pawlita have received research support through cooperation contracts of DKFZ with Roche and Qiagen in the field of development of HPV diagnostics.

\section{Authors' contributions}

LB carried out the molecular analyses and drafted the manuscript, MP conceived the study, participated in its design and coordination, and supervised to draft the manuscript, PBR, GS, GT, RB and MCDM participated in the management of the patient, provided the samples and contributed to draft the clinical part of the manuscript, ADM contributed and supervised to draft the manuscript, DH supervised the molecular analyses and contributed to draft the manuscript, LB and RB performed the TP53 sequencing analyses, AM provided the serological data. MS contributed to draft the manuscript. LA provided unpublished metanalyses data on HPV type 58. All authors read and approved the final manuscript.

\section{Acknowledgements}

This study was funded in part by European Commission grant HPV-AHEAD (FP7-HEALTH-2011-282562 (to M. Pawlita and D. Holzinger) and in part by Lega Italiana per la Lotta contro I Tumori (LILT). We appreciate the excellent technical assistance of A. Leischwitz, U. Koch and M. Oppenländer.

\section{Author details}

'Division of Genome Modifications and Carcinogenesis (F020), Research Program Infection and German Cancer Research Center (DKFZ), ImNeuenheimer Feld 280, Heidelberg 69120, Germany. ${ }^{2}$ Department of Surgery, Oncology and Gastroenterology, Immunology and Oncology Section, University of Padua, Via Gattamelata, 64, Padua 35128-I, Italy. ${ }^{3}$ Veneto Institute of Oncology IOV - IRCCS, Immunology and Molecular Oncology Unit, Via Gattamelata, 64, Padua 35128-I, Italy. ${ }^{4}$ Department of Neurosciences, ENT Clinic and Regional Center for Head and Neck Cancer, University of Padua, School of Medicine, Treviso Regional Hospital, Piazzale Ospedale 1, Treviso 31100, Italy. ${ }^{5}$ Head and Neck Department, Hospital of Cattinara, University of Trieste, Strada di Fiume 447, Trieste 34149, Italy. ${ }^{6}$ Pathology Department, Hospital of Cattinara, University of Trieste, Strada di Fiume 447, Trieste 34149, Italy. ${ }^{7}$ IDIBELL, Institut Catalàd' Oncologia - Catalan Institute of Oncology, Avda Gran Via de I'Hospitalet, Barcelona 199-203, Spain.

Received: 29 August 2013 Accepted: 6 November 2013 Published: 12 November 2013

\section{References}

1. van Monsjou HS, Balm AJ, van den Brekel MM, Wreesmann VB: Oropharyngeal squamous cell carcinoma: a unique disease on the rise? Oral Oncol 2010, 46:780-785.

2. Ferlay J, Shin HR, Bray F, Forman D, Mathers C, Parkin DM: Estimates of worldwide burden of cancer in, GLOBOCAN 2008. Int J Cancer 2010, 127:2893-2917.

3. Globocan; 2008. http://globocan.iarc.fr/.

4. Anantharaman D, Gheit T, Waterboer T, Abedi-Ardekani B, Carreira C, McKay-Chopin S, Gaborieau V, Marron M, Lagiou P, Ahrens W, et al:
Human papillomavirus infections and upper aero-digestive tract cancers: the ARCAGE study. J Nat/ Cancer Inst 2013, 105:536-545.

5. Chaturvedi AK, Engels EA, Pfeiffer RM, Hernandez BY, Xiao W, Kim E, Jiang B, Goodman MT, Sibug-Saber M, Cozen W, et al: Human papillomavirus and rising oropharyngeal cancer incidence in the United States. J Clin Oncol 2011, 29:4294-4301.

6. IARC: Human papillomaviruses. IARC Monogr Eval Carcinog Risks Hum 2011; 100B: 1-475. A review of human carcinogens_-Part B: biological agents, vol. 100B, 2011. IARC Monogr Eval Carcinog Risks Hum 2011, 100:1-475.

7. de Sanjose S, Quint WG, Alemany L, Geraets DT, Klaustermeier JE, Lloveras B, Tous S, Felix A, Bravo LE, Shin HR, et al: Human papillomavirus genotype attribution in invasive cervical cancer: a retrospective cross-sectional worldwide study. Lancet Oncol 2010, 11:1048-1056.

8. Holzinger D, Schmitt M, Dyckhoff G, Benner A, Pawlita M, Bosch FX: Viral RNA patterns and high viral load reliably define oropharynx carcinomas with active HPV16 involvement. Cancer Res 2012, 72:4993-5003.

9. Kreimer AR, Clifford GM, Boyle P, Franceschi S: Human papillomavirus types in head and neck squamous cell carcinomas worldwide: a systematic review. Cancer Epidemiol Biomarkers Prev 2005, 14:467-475.

10. St Guily JL, Jacquard AC, Pretet JL, Haesebaert J, Beby-Defaux A, Clavel C, Agius G, Birembaut $P$, Okais $C$, Leocmach $Y$, et al: Human papillomavirus genotype distribution in oropharynx and oral cavity cancer in France-The EDiTH VI study. J Clin Virol 2011, 51:100-104.

11. Huang $H$, Zhang B, Chen W, Zou SM, Zhang YX, Qiao YL: Detection of human papillomavirus in oropharyngeal squamous cell carcinoma. Zhongguo Yi Xue Ke Xue Yuan Xue Bao 2012, 34:545-549.

12. Kato H, Yui T, Yamamoto N, Okada T, Urano M, Sakurai K, Naito K: The clinical significance of thymidylate synthase expression in human papillomavirus-related oropharyngeal squamous carcinoma. Nihon Jibiinkoka Gakkai Kaiho 2012, 115:957-964.

13. Paolini F, Rizzo C, Sperduti I, Pichi B, Mafera B, Rahimi SS, Vigili MG, Venuti A: Both mucosal and cutaneous papillomaviruses are in the oral cavity but only alpha genus seems to be associated with cancer. J Clin Virol 2013, 56:72-76.

14. Kuo KT, Hsiao CH, Lin CH, Kuo LT, Huang SH, Lin MC: The biomarkers of human papillomavirus infection in tonsillar squamous cell carcinoma-molecular basis and predicting favorable outcome. Mod Pathol 2008, 21:376-386.

15. Kim SH, Koo BS, Kang S, Park K, Kim H, Lee KR, Lee MJ, Kim JM, Choi EC, Cho NH: HPV integration begins in the tonsillar crypt and leads to the alteration of p16, EGFR and c-myc during tumor formation. Int J Cancer 2007, 120:1418-1425.

16. D'Souza G, Kreimer AR, Viscidi R, Pawlita M, Fakhry C, Koch WM, Westra WH, Gillison ML: Case-control study of human papillomavirus and oropharyngeal cancer. N Engl J Med 2007, 356:1944-1956.

17. Heideman DA, Waterboer T, Pawlita M, Delis-van Diemen P, Nindl I, Leijte JA, Bonfrer JM, Horenblas S, Meijer CJ, Snijders PJ: Human papillomavirus-16 is the predominant type etiologically involved in penile squamous cell carcinoma. J Clin Oncol 2007, 25:4550-4556.

18. Herrero $R$, Castellsague X, Pawlita M, Lissowska J, Kee F, Balaram P, Rajkumar T, Sridhar H, Rose B, Pintos J, et al: Human papillomavirus and oral cancer: the International Agency for Research on Cancer multicenter study. J Natl Cancer Inst 2003, 95:1772-1783.

19. Liang C, Marsit CJ, McClean MD, Nelson HH, Christensen BC, Haddad Rl, Clark JR, Wein RO, Grillone GA, Houseman EA, et al: Biomarkers of HPV in head and neck squamous cell carcinoma. Cancer Res 2012, 72:5004-5013.

20. Meschede W, Zumbach K, Braspenning J, Scheffner M, Benitez-Bribiesca L, Luande J, Gissmann L, Pawlita M: Antibodies against early proteins of human papillomaviruses as diagnostic markers for invasive cervical cancer. J Clin Microbiol 1998, 36:475-480.

21. Ribeiro KB, Levi JE, Pawlita M, Koifman S, Matos E, Eluf-Neto J, Wunsch-Filho V, Curado MP, Shangina O, Zaridze D, et al: Low human papillomavirus prevalence in head and neck cancer: results from two large case-control studies in high-incidence regions. Int J Epidemiol 2011, 40:489-502.

22. Smeets SJ, Hesselink AT, Speel EJ, Haesevoets A, Snijders PJ, Pawlita M, Meijer CJ, Braakhuis BJ, Leemans CR, Brakenhoff RH: A novel algorithm for reliable detection of human papillomavirus in paraffin embedded head and neck cancer specimen. Int J Cancer 2007, 121:2465-2472.

23. Zumbach K, Hoffmann M, Kahn T, Bosch F, Gottschlich S, Gorogh T, Rudert H, Pawlita M: Antibodies against oncoproteins E6 and E7 of human papillomavirus types 16 and 18 in patients with head-and-neck squamous-cell carcinoma. Int J Cancer 2000, 85:815-818. 
24. Zumbach K, Kisseljov F, Sacharova O, Shaichaev G, Semjonova L, Pavlova L, Pawlita M: Antibodies against oncoproteins E6 and E7 of human papillomavirus types 16 and 18 in cervical-carcinoma patients from Russia. Int J Cancer 2000, 85:313-318.

25. Halec G, Schmitt M, Dondog B, Sharkhuu E, Wentzensen N, Gheit T, Tommasino M, Kommoss F, Bosch FX, Franceschi S, et al: Biological activity of probable/possible high-risk human papillomavirus types in cervical cancer. Int J Cancer 2013, 132:63-71.

26. Holzinger D, Flechtenmacher C, Henfling N, Kaden I, Grabe N, Lahrmann B, Schmitt M, Hess J, Pawlita M, Bosch FX: Identification of oropharyngeal squamous cell carcinomas with active HPV16 involvement by immunohistochemical analysis of the retinoblastoma protein pathway. Int J Cancer 2013, 133:1389-1399.

27. Halec G, Holzinger D, Schmitt M, Flechtenmacher C, Dyckhoff G, Lloveras B, Hofler D, Bosch FX, Pawlita M: Biological evidence for a causal role of HPV16 in a small fraction of laryngeal squamous cell carcinoma. Br J Cancer 2013, 109:172-183.

28. Lingen MW, Xiao W, Schmitt A, Jiang B, Pickard R, Kreinbrink $P$, Perez-Ordonez B, Jordan RC, Gillison ML: Low etiologic fraction for high-risk human papillomavirus in oral cavity squamous cell carcinomas. Oral Oncol 2013, 49:1-8.

29. Machalek DA, Poynten M, Jin F, Fairley CK, Farnsworth A, Garland SM, Hillman RJ, Petoumenos K, Roberts J, Tabrizi SN, et al: Anal human papillomavirus infection and associated neoplastic lesions in men who have sex with men: a systematic review and meta-analysis. Lancet Oncol 2012, 13:487-500.

30. de Koning MN, Quint WG, Pirog EC: Prevalence of mucosal and cutaneous human papillomaviruses in different histologic subtypes of vulvar carcinoma. Mod Pathol 2008, 21:334-344.

31. Schmitt M, Bravo IG, Snijders PJ, Gissmann L, Pawlita M, Waterboer T: Bead-based multiplex genotyping of human papillomaviruses. J Clin Microbio/ 2006, 44:504-512.

32. Schmitt M, Depuydt C, Benoy I, Bogers J, Antoine J, Arbyn M, Pawlita M: Prevalence and viral load of 51 genital human papillomavirus types and three subtypes. Int J Cancer 2013, 132:2395-2403.

33. Schmitt M, Dondog B, Waterboer T, Pawlita M: Homogeneous amplification of genital human alpha papillomaviruses by PCR using novel broad-spectrum GP5+ and GP6+ primers. J Clin Microbiol 2008, 46:1050-1059.

34. Boscolo-Rizzo P, Da Mosto MC, Fuson R, Frayle-Salamanca H, Trevisan R, Del Mistro A: HPV-16 E6 L83V variant in squamous cell carcinomas of the upper aerodigestive tract. J Cancer Res Clin Oncol 2009, 135:559-566.

35. Sehr P, Muller M, Hopfl R, Widschwendter A, Pawlita M: HPV antibody detection by ELISA with capsid protein L1 fused to glutathione S-transferase. J Virol Methods 2002, 106:61-70.

36. Sehr P, Zumbach $K$, Pawlita M: A generic capture ELISA for recombinant proteins fused to glutathione S-transferase: validation for HPV serology. J Immunol Methods 2001, 253:153-162.

37. Waterboer T, Sehr P, Michael KM, Franceschi S, Nieland JD, Joos TO, Templin MF, Pawlita M: Multiplex human papillomavirus serology based on in situ-purified glutathione s-transferase fusion proteins. Clin Chem 2005, 51:1845-1853.

38. Waterboer T, Sehr P, Pawlita M: Suppression of non-specific binding in serological Luminex assays. J Immunol Methods 2006, 309:200-204.

39. IARC: TP53 direct sequencing protocol; 2010. http://p53.iarc.fr/Download/ TP53_DirectSequencing_IARC.pdf.

40. Ang KK, Harris J, Wheeler R, Weber R, Rosenthal DI, Nguyen-Tan PF, Westra WH, Chung $\mathrm{CH}$, Jordan RC, Lu C, et al: Human papillomavirus and survival of patients with oropharyngeal cancer. N Engl J Med 2010, 363:24-35.

41. Fakhry C, Westra WH, Li S, Cmelak A, Ridge JA, Pinto H, Forastiere A, Gillison ML: Improved survival of patients with human papillomavirus-positive head and neck squamous cell carcinoma in a prospective clinical trial. J Natl Cancer Inst 2008, 100:261-269.

42. Lechner M, Frampton GM, Fenton $T$, Andrew F, Palmer G, Jay A, Pillay $N$ Forster M, Cronin MT, Lipson D, et al: Targeted next-generation sequencing of head and neck squamous cell carcinoma identifies novel genetic alterations in HPV+ and HPV-tumors. Genome Medicine 2013, 5:49.

43. Agrawal N, Frederick MJ, Pickering CR, Bettegowda C, Chang K, Li RJ, Fakhry C, Xie TX, Zhang J, Wang J, et al: Exome sequencing of head and neck squamous cell carcinoma reveals inactivating mutations in NOTCH1. Science 2011, 333:1154-1157.
44. Stransky N, Egloff AM, Tward AD, Kostic AD, Cibulskis K, Sivachenko A, Kryukov GV, Lawrence MS, Sougnez C, McKenna A, et al: The mutational landscape of head and neck squamous cell carcinoma. Science 2011, 333:1157-1160.

45. Jung AC, Briolat J, Millon R, de Reynies A, Rickman D, Thomas E, Abecassis J, Clavel C, Wasylyk B: Biological and clinical relevance of transcriptionally active human papillomavirus (HPV) infection in oropharynx squamous cell carcinoma. Int J Cancer 2010, 126:1882-1894.

46. van Houten VM, Snijders PJ, van den Brekel MW, Kummer JA, Meijer CJ, van Leeuwen B, Denkers F, Smeele LE, Snow GB, Brakenhoff RH: Biological evidence that human papillomaviruses are etiologically involved in a subgroup of head and neck squamous cell carcinomas. Int J Cancer 2001, 93:232-235.

47. Wiest T, Schwarz E, Enders C, Flechtenmacher C, Bosch FX: Involvement of intact HPV16 E6/E7 gene expression in head and neck cancers with unaltered p53 status and perturbed pRb cell cycle control. Oncogene 2002, 21:1510-1517.

48. Li Y, Wang X, Ni T, Wang F, Lu W, Zhu J, Xie X, Zheng ZM: Human papillomavirus type 58 genome variations and RNA expression in cervical lesions. J Virol 2013, 87:9313-9322.

\section{doi:10.1186/1743-422X-10-334}

Cite this article as: Baboci et al.: Evidence of the causal role of human papillomavirus type 58 in an oropharyngeal carcinoma. Virology Journal $201310: 334$

\section{Submit your next manuscript to BioMed Central and take full advantage of:}

- Convenient online submission

- Thorough peer review

- No space constraints or color figure charges

- Immediate publication on acceptance

- Inclusion in PubMed, CAS, Scopus and Google Scholar

- Research which is freely available for redistribution 